\title{
Plug potential formation in a tandem mirror
}

\author{
I. Katanuma, ${ }^{\text {a) }}$ Y. Kiwamoto, Y. Tatematsu, K. Ishii, T. Saito, K. Yatsu, and T. Tamano \\ Plasma Research Center, University of Tsukuba, Ibaraki 305, Japan
}

(Received 5 November 1996; accepted 6 March 1997)

It is suggested experimentally that a plug potential forms in a different mechanism from the originally expected scenario in the present tandem mirror [T. Tamano, Phys. Plasmas 2, 2321 (1995)]. That is, the formation requires only electron cyclotron resonance heating experimentally. In this manuscript, therefore, the plug potential with continuous axial profile is analytically shown to be created with the plausible ion and electron distribution functions in a present tandem mirror, i.e., passing ions, mirror trapped ions in the thermal barrier, passing electrons, $\phi$-trapped electrons in a plug potential, and the small amount of ions that are born around the plug region. (C) 1997 American Institute of Physics. [S1070-664X(97)01406-7]

\section{INTRODUCTION}

In order to improve the severe technological requirements of the plug in the original tandem mirror, ${ }^{1,2}$ the idea of the thermal barrier was proposed. ${ }^{3}$ The subsequent tandem mirror experimental devices adopted the thermal barrier, where the plug potential is created with the help of sloshing ions. ${ }^{4}$

The sloshing ions are made by neutral beam injection (NBI). The resultant ion density hill at the plug leads to the local potential maximum there. The electrons heated by electron cyclotron resonance heating (ECRH) around the plug make the potential high enough to confine the central cell ions in a tandem mirror. So the plug potential formation requires both $\mathrm{NBI}$ and ECRH in the original scenario.

Subsequent experiments, however, found that the ionconfining potential can be created independently of the neutral beam injection. Namely, the plug potential was made by ECRH alone. These observations were reported on two different experimental devices. ${ }^{5,6}$

Theoretical efforts to understand the plug potential formation have been made. ${ }^{7-11}$ After the formation of a plug potential was observed experimentally with ECRH only in a tandem mirror, the main results were that a maximum position of the electrostatic potential locates at an outer mirror throat. The experimental measurements of the potential profile, however, have been carried out at only a single point in each mirror cell until now. ${ }^{12-14}$ So it remains unknown experimentally where the maximum point of electrostatic potential (plug potential) exists.

Recently, the potential around the outer mirror throat of the GAMMA 10 tandem mirror ${ }^{5}$ was measured with Langmuir probes, the results and analysis of which indicate that the maximum point of the potential exists between the outer mirror throat $\left[z=z_{m}\right.$ in Fig. 1(a)] and the thermal barrier region $\left(z=z_{b}\right)$, rather than just at the outer mirror throat. ${ }^{15-17}$

The purpose of this manuscript is to examine a possible mechanism of the plug potential formation in a tandem mirror with ECRH alone. Here, we define the terminology "plug region" as the region where the electrostatic potentiale is the highest [i.e., $z=z_{p}$ in Fig. 1(a)] between $z=z_{b}$

${ }^{a)}$ Electronic mail: katanuma@prc.tsukuba.ac.jp and $z=z_{m}$, and define the "plug potential" as the highest electrostatic potential at $z=z_{p}$.

\section{MODEL OF ION AND ELECTRON DISTRIBUTION FUNCTIONS}

At first we consider the ion distribution function in the region $z \leqslant z_{p}$. Here, the ion velocity space diagram written by energy $\epsilon$ and magnetic moment $\mu$ is seen in Fig. 1(b). The ion distribution function $f_{i}$ is assumed as follows in the region of $v_{\|} \geqslant 0$,

$$
\begin{aligned}
f_{i} & =n_{i c}\left(\frac{m_{i}}{2 \pi T_{i}}\right)^{3 / 2} \exp \left\{-\frac{\epsilon-e \phi_{i}}{T_{i}}\right\} \quad \text { for } \epsilon \geqslant \mu B_{i}+e \phi_{i}, \\
f_{i} & =n_{i c}\left(\frac{m_{i}}{2 \pi T_{i}}\right)^{3 / 2} \exp \left\{-\frac{\epsilon-e \phi_{i}-\alpha_{i} \mu B_{i}}{\left(1-\alpha_{i}\right) T_{i}}\right\} \text { for } \epsilon \\
& <\mu B_{i}+e \phi_{i},
\end{aligned}
$$

while in the region of $v_{\|}<0$,

$$
\begin{aligned}
f_{i}= & n_{i c}\left(\frac{m_{i}}{2 \pi T_{i}}\right)^{3 / 2} \exp \left\{-\frac{\epsilon-e \phi_{i}}{T_{i}}\right\} \quad \text { for } \epsilon \geqslant \mu B_{i} \\
& +e \phi_{i} \text { and }\left(\epsilon<\mu B_{m}+e \phi_{m} \text { or } \epsilon<\mu B_{p}+e \phi_{p}\right) \\
f_{i}= & n_{i p}\left(\frac{m_{i}}{2 \pi T_{i}}\right)^{3 / 2} \exp \left\{-\frac{\epsilon-e \phi_{p}-\alpha_{p} \mu B_{p}}{\left(1-\alpha_{p}\right) T_{i}}\right\} \quad \text { for } \epsilon \\
\geqslant & \mu B_{p}+e \phi_{p} \text { and } \mu<\mu_{*}, \\
f_{i} & =n_{i m}\left(\frac{m_{i}}{2 \pi T_{i}}\right)^{3 / 2} \exp \left\{-\frac{\epsilon-e \phi_{m}-\alpha_{p} \mu B_{m}}{\left(1-\alpha_{p}\right) T_{i}}\right\} \text { for } \epsilon \\
& \geqslant \mu B_{m}+e \phi_{m} \quad \text { and } \mu \geqslant \mu_{*}, \\
f_{i} & =n_{i c}\left(\frac{m_{i}}{2 \pi T_{i}}\right)^{3 / 2} \exp \left\{-\frac{\epsilon-e \phi_{i}-\alpha_{i} \mu B_{i}}{\left(1-\alpha_{i}\right) T_{i}}\right\} \text { for } \epsilon \\
& <\mu B_{i}+e \phi_{i}, \quad
\end{aligned}
$$

where $v_{\|}$is the ion velocity along the magnetic-field line, $n_{i p}=n_{i c} \exp \left\{-e\left[\phi_{p}-\phi_{i}\right] / T_{i}\right\}, \quad$ and $n_{i m}=n_{i c} \exp \left\{-e\left[\phi_{m}\right.\right.$ $\left.\left.-\phi_{i}\right] / T_{i}\right\}$. Here, $\mu_{*} \equiv-\left(e \phi_{p}-e \phi_{m}\right) /\left(B_{p}-B_{m}\right)$ is the value of $\mu$ at the intersection of two lines $\epsilon=\mu B_{p}+e \phi_{p}, \epsilon$ $=\mu B_{m}+e \phi_{m}$ in Fig. 1(b). Henceforth, the standard nota- 

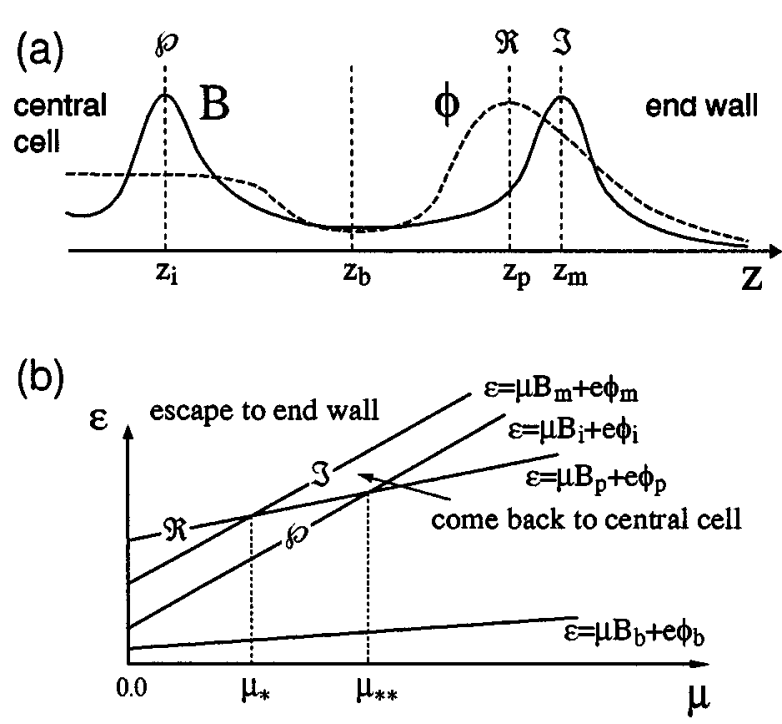

FIG. 1. Schematic diagram of the end mirror cell of a tandem mirror. (a) is the axial magnetic and electrostatic potential profiles. (b) is the ion velocity space described by $\epsilon$ and $\mu$. Here, the symbols $\wp, \mathfrak{R}$, and $\mathfrak{F}$ are the lines $\epsilon=\mu B_{i}+e \phi_{i}, \epsilon=\mu B_{p}+e \phi_{p}, \epsilon=\mu B_{m}+e \phi_{m}$, respectively, in (b). These lines symbolized by $\wp, \mathfrak{R}$, and $\mathfrak{F}$ in (b) correspond to the ion velocities, the parallel component of which, $v_{\|}$, is zero at $z=z_{i}, z=z_{p}$, and $z=z_{m}$, respectively in (a).

tions are used. The suffixes $i, m$, and $p$ of field quantities are the values at $z=z_{i}, z=z_{m}$, and $z=z_{p}$ defined in Fig. 1(a), respectively.

The ions flowing out of the central cell of a tandem mirror are assumed to be Maxwellian (in the range $v_{\|} \geqslant 0$ ) at the inner mirror throat $\left(z=z_{i}\right)$. The ions trapped in the thermal barrier region are assumed to be non-Maxwellian because the ion density at $z=z_{b}$ is usually smaller than that in the central cell when the nonzero thermal barrier potential depth is observed, which was pointed out experimentally by Post $e t a l .{ }^{6}$ The ion distribution functions are different from each other in $v_{\|} \geqslant 0$ and $v_{\|}<0$ in Eqs. (1)-(6), because the ions passing over the outer mirror throat $\left(z=z_{m}\right)$ do not come back to the thermal barrier region.

The ion distribution function in the region $z_{m} \geqslant z>z_{p}$ is given as follows: At first, the ion distribution function $f_{i}$ is assumed in the region of $v_{\|} \geqslant 0$, which is

$$
\begin{aligned}
f_{i}= & n_{i c}\left(\frac{m_{i}}{2 \pi T_{i}}\right)^{3 / 2} \exp \left\{-\frac{\epsilon-e \phi_{i}}{T_{i}}\right\} \text { for } \epsilon \geqslant \mu B_{i} \\
& +e \phi_{i} \text { and } \epsilon \geqslant \mu B_{p}+e \phi_{p},
\end{aligned}
$$

where these ions flow from the central cell. The distribution function of ions trapped in the region $z_{m} \geqslant z>z_{p}$ is given, respectively, in each region of velocity space as

$$
\begin{aligned}
f_{i} & =n_{i c}\left(\frac{m_{i}}{2 \pi T_{i}}\right)^{3 / 2} \exp \left\{-\frac{\epsilon-e \phi_{i}-\alpha_{i} \mu B_{i}}{\left(1-\alpha_{i}\right) T_{i}}\right\} \text { for } \epsilon \\
& <\mu B_{i}+e \phi_{i} \text { and } \epsilon \geqslant \mu B_{p}+e \phi_{p},
\end{aligned}
$$

$$
\begin{aligned}
f_{i}= & n_{i c}\left(\frac{m_{i}}{2 \pi T_{i}}\right)^{3 / 2} \exp \left\{\frac{e \phi_{p}-e \phi_{i}}{\left(\alpha_{i}-1\right) T_{i}}\right\} \\
& \times \exp \left\{-\frac{\epsilon-e \phi_{p}-\alpha_{y} \mu B_{p}}{\left(1-\alpha_{y}\right) T_{y}}\right\} \\
& \text { for } \epsilon<\mu B_{p}+e \phi_{p} \quad \text { and } \mu \geqslant \mu_{* *}, \\
f_{i}= & n_{i p}\left(\frac{m_{i}}{2 \pi T_{i}}\right)^{3 / 2} \exp \left\{-\frac{\epsilon-e \phi_{p}-\alpha_{x} \mu B_{p}}{\left(1-\alpha_{x}\right) T_{i}}\right\} \\
& \text { for } \epsilon<\mu B_{p}+e \phi_{p} \quad \text { and } \mu_{* *}>\mu \geqslant \mu_{*}, \\
f_{i}= & n_{i p}\left(\frac{m_{i}}{2 \pi T_{i}}\right)^{3 / 2} \exp \left\{-\frac{\epsilon-e \phi_{p}-\alpha_{x} \mu B_{p}}{\left(1-\alpha_{x}\right) T_{i}}\right\} \\
& \text { for } \epsilon<\mu B_{m}+e \phi_{m} \quad \text { and } \mu<\mu_{*}, \\
f_{i}= & n_{i p}\left(\frac{m_{i}}{2 \pi T_{i}}\right)^{3 / 2} \exp \left\{-\frac{\epsilon-e \phi_{p}-\alpha_{q} \mu B_{p}}{\left(1-\alpha_{q}\right) T_{i}}\right\}
\end{aligned}
$$

for $\epsilon<\mu B_{p}+e \phi_{p}$ and $\epsilon \geqslant \mu B_{m}+e \phi_{m} \quad$ and $\mu<\mu_{*}$.

Here, $\mu_{* *} \equiv-\left(e \phi_{p}-e \phi_{i}\right) /\left(B_{p}-B_{i}\right)$ is the magnitude of $\mu$ at the intersection of the lines $\epsilon=\mu B_{p}+e \phi_{p}$ and $\epsilon=\mu B_{i}$ $+e \phi_{i}$. The constants $T_{y}, \alpha_{y}, \alpha_{x}$, and $\alpha_{q}$ satisfy the relations in order that the distribution function is continuous across the separatrix of each region in Eqs. (8), (9), and (10), i.e., $\left(\alpha_{i} B_{i} / B_{p}-1\right) T_{y}=\left(\alpha_{i}-1\right) T_{i}$, where the distribution functions (8) and (9) are continuous across the line $\mathfrak{R}$ in Fig. $1(\mathrm{~b})$, and $\left(\alpha_{x}-1\right) T_{i}=\left(\alpha_{y}-1\right) T_{y}$, where the distribution functions (9) and (10) are continuous across the line $\mu$ $=\mu_{* *}$.

The electron distribution function $f_{e}$ is assumed to be

$$
\begin{aligned}
f_{e}= & n_{e b}\left(\frac{m_{e}}{2 \pi T_{e}}\right)^{3 / 2} \exp \left\{-\frac{\epsilon+e \phi_{b}}{T_{e}}\right\} \text { for } \epsilon \geqslant \mu B_{b} \\
& -e \phi_{b}, \\
f_{e} & =n_{e b}\left(\frac{m_{e}}{2 \pi T_{e}}\right)^{3 / 2} \exp \left\{-\frac{\epsilon+e \phi_{b}-\alpha_{e} \mu B_{b}}{\left(1-\alpha_{e}\right) T_{e}}\right\} \text { for } \epsilon \\
& <\mu B_{b}-e \phi_{b},
\end{aligned}
$$

where $n_{e b}=n_{i c} \exp \left\{e\left[\phi_{b}-\phi_{i}\right] / T_{e}\right\}$. Here, the electron distribution function (13) and (14) is applied in the region $z_{b} \leqslant z$ $\leqslant z_{m}$ in Fig. 1(a). The electrons are assumed to be Maxwellian in a part that comes from the central cell. The fundamental electron cyclotron resonance heating $\left(\omega_{\mathrm{ce}} \mathrm{ECRH}\right)$ is assumed to be applied at the point $B=2 B_{b}$, so that the electrons trapped in the plug potential are assumed to be the non-Maxwellian distribution in Eq. (14), which is consistent with the Fokker-Planck simulation results obtained by Matsuda and Rognlien. ${ }^{18}$

The parameters $\alpha_{i}, \alpha_{x}, \alpha_{p}, \alpha_{q}$, and $\alpha_{e}$ describe the degree to which the trapped-particle phase space is filled, as adopted by Cohen. ${ }^{19}$ This type of distribution function is a simplest distribution function, which has the effect of the non-Maxwellian distribution but is analytically integrable in velocity space. Here, we require that $0 \leqslant \alpha_{p} \leqslant 1$ in Eqs. (4) and (5), assuming that ions are not produced in the region 
outside the outer mirror throat. And, we require that $\alpha_{q} \geqslant 1$ in Eq. (12), where $\alpha_{q}$ should have the value close to unity.

Here, we show the various orbits of ions and electrons that are taken into account in this manuscript. Figure 2(a) displays the schematic diagram of orbits in the axial magnetic and potential profiles shown in Fig. 2(b). The orbit symbolized by (1) is that of ions that come from the central cell, where the distribution function is given by Eq. (1). The orbit (2) is that of ions that are trapped magnetically in the end mirror cell, where these ions are given in Eq. (8). The symbol (3) is the ion orbit where ions are trapped electrostatically in the end mirror cell and the distribution function is given by Eqs. (2) and (6). The Yushmanov trapped ions are given by the orbits (4), (5), and (6), where ions are trapped in the region between $z=z_{p}$ and $z=z_{m}$. The distribution functions with orbits of the Yushmanov trapped ions (4), (5), and (6) are given by Eqs. (9), (10), and (11), respectively. The orbits of ions that are born around the plug region are (7) and (8). The distributions functions with orbits (7) and (8) are given by Eqs. (4), (5), and (12), respectively.

The electron orbits are indicated by the symbols (a), (b). The electrons flowing from the central cell are reflected first by the thermal barrier potential depth, and second by the potential drop beyond the outer mirror throat, where the distribution function is given by Eq. (13). The electrons trapped in the plug potential are denoted by the symbol (b), and its distribution function is given by Eq. (14).

The ion velocity space $(\epsilon, \mu)$ diagram is shown in Fig. 2(c). Here, the axial region $z_{p} \leqslant z \leqslant z_{m}$ is considered in Fig. 2 (c). The location of each symbol displays the region of existence in the $\epsilon, \mu$ space, where the ions in the region with each symbol in Fig. 2(c) correspond to those with the orbit indicated by the same symbol in Fig. 2(a). The distribution function should be continuous everywhere in the velocity space, so that the distribution functions defined in each region (1)-(8) are required to be continuous across the boundaries of the region where the distribution functions are defined.

The Yushmanov trapped ions in regions (4), (5), and (6) exist in a different region from each other in Fig. 2(c). The distribution function in (4) is continuous with that in (2) across the separatrix $\mathfrak{R}$, and the distribution function in (5) should be joined continuously to that in (1) across the line $\mathfrak{R}$. So the distribution functions in (4) and (5) are defined with different forms, as seen in Eqs. (9) and (10).

Whether the jump of the potential (formation of sheath potential) occurs across the axial coordinate $z=z_{p}$ depends on whether the ion distribution function is continuous across line $\mathfrak{R}$ in the velocity space in Fig. 2(c), as shown later in this manuscript. The distribution function of ionized ions in region (8) in Fig. 2(c), therefore, is required to be continuous with that in (1) across the separatrix $\mathfrak{R}$. And the coefficient $\alpha_{q}$ in Eq. (12) is $\alpha_{q} \geqslant 1$ in order that the distribution function of ionized ions decreases away from line $\mathfrak{R}$.

The distribution function of ions in region (2) is the same as that of ions in (3) in Fig. 2(c). As mentioned previously in this section, the ion distribution functions trapped in the ther- (a)
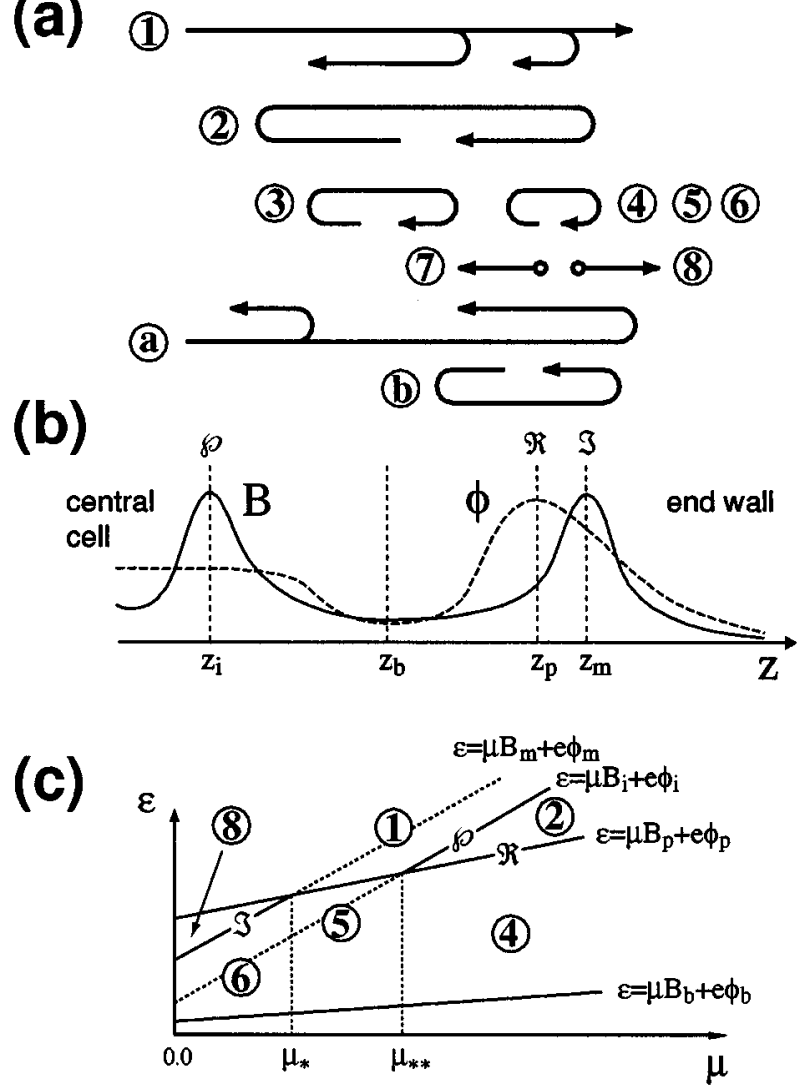

FIG. 2. Schematic diagram of ion and electron orbits: (a) is the orbits in the magnetic field and potential profiles, which are shown in (b), and (c) is the ion velocity $(\epsilon, \mu)$ space diagram.

mal barrier region are assumed to be non-Maxwellian, where the constant $\alpha_{i}$ is introduced in Eqs. (2), (6), and (8). Similarly, the Yushmanov trapped ions are also assumed to be non-Maxwellian. So, we introduce a constant $\alpha_{x}$ in the distribution function of Eqs. (10) and (11).

Although the ion distribution function in Eq. (9), which is symbolized by (4) in Figs. 2(a) and 2(c), has constants $T_{y}$ and $\alpha_{y}$, these constants are determined uniquely in terms of $T_{i}, \alpha_{i}$, and $\alpha_{x}$ because of the continuity condition of the distribution function across the line $\mu=\mu_{* *}$ and the line $\mathfrak{R}$, as already mentioned in this section.

The Yushmanov trapped ions symbolized by (4), (5), and (6) in Fig. 2 do not influence the formation of plug potential, but have an effect on the potential profile from $z=z_{p}$ to $z$ $=z_{m}$.

Examples of the ion distribution function at $z=z_{b}$ are displayed in Fig. 3. The ion distribution function in the region $v_{\|} \geqslant 0$ is different from that in the region $v_{\|}<0$ at $z$ $=z_{b}$ in Fig. 2 because the ions escaping from the outer mirror throat $z=z_{m}$ are assumed to not come back to the central cell. The ion distribution function in Eq. (2) is written in terms of $v_{\|}$and $v_{\perp}$ at $z=z_{b}$, which reduces to

$$
f_{i}=n_{i c}\left(\frac{m_{i}}{2 \pi T_{i}}\right)^{3 / 2} \exp \left\{-\frac{m_{i} v_{\|}^{2}}{2 T_{\|}}-\frac{m_{i} v_{\perp}^{2}}{2 T_{\perp}}-\frac{e \phi_{b}-e \phi_{i}}{\left(1-\alpha_{i}\right) T_{i}}\right\},
$$

with $T_{\|}=\left(1-\alpha_{i}\right) T_{i}$ and $T_{\perp}=\left(1-\alpha_{i}\right) T_{i} /\left(1-\alpha_{i} B_{i} / B_{b}\right)$ by using the relations $\epsilon=\frac{1}{2} m_{i}\left(v_{\|}^{2}+v_{\perp}^{2}\right)+e \phi_{b}$ and $\mu$ 

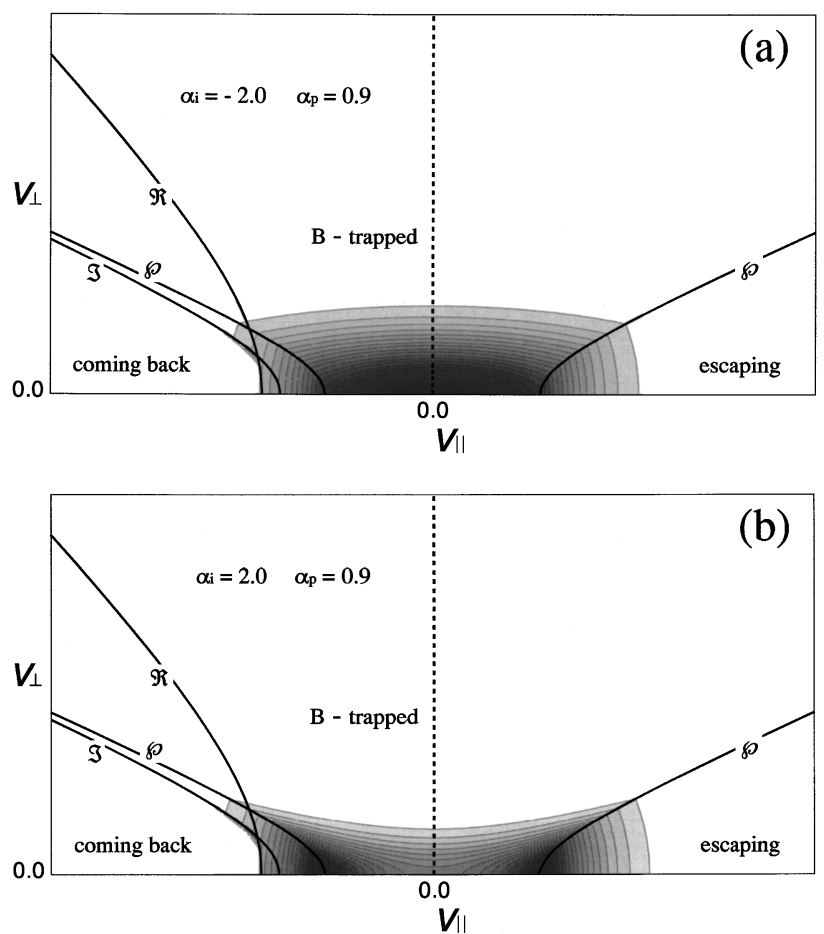

FIG. 3. The contour plot of ion distribution function at the thermal barrier $z=z_{b}$. In the figure, the parameters are $B_{p} / B_{b}=2.0, B_{i} / B_{b}=B_{m} / B_{b}$ $=6.0, e\left(\phi_{i}-\phi_{b}\right) / T_{i}=1.0, e\left(\phi_{p}-\phi_{i}\right) / T_{i}=1.5$, and $e\left(\phi_{m}-\phi_{i}\right) / T_{i}=1.0$, respectively. The solid lines describe the separatrixes. The lines denoted by $\wp, \mathfrak{R}$, and $\mathfrak{F}$ correspond to the lines with the same symbols in Fig. 1(b); (a) is $\alpha_{i}=-2.0, \alpha_{p}=0.9$, and (b) is $\alpha_{i}=2.0, \alpha_{p}=0.9$.

$=m_{i} v_{\perp}^{2} / 2 B_{b}$. The distribution becomes bi-Maxwellian with positive $T_{\|}$and $T_{\perp}$ in the case of $\alpha_{i}<0$ in the region indicated by " $B$-trapped" in Fig. 3(a). The distribution function, however, changes from Maxwellian to the state of no trapped ions in the case of $\alpha_{i}>1$ in Fig. 3(b).

Examples of the electron distribution function at $z=z_{p}$ are shown in Fig. 4. The electron distribution function is symmetric about $v_{\|}=0$, with the assumption, in this manuscript that all electrons escaping from the outer mirror throat come back to the central cell again, so that only the region $v_{\|} \geqslant 0$ is displayed in Figs. 4(b) and 4(d). The passing electrons are assumed to be Maxwellian, which is given in Eq. (13), and the passing component of electrons is seen in the region denoted by "passing" in Figs. 4(b) and 4(d) and in the region $\epsilon \geqslant \mu B_{b}-e \phi_{b}$ in Figs. 4(a) and 4(c). The electron distribution function in Eq. (14) is the same type of ions as in Eq. (2). The distribution function of electrons trapped in the plug potential becomes bi-Maxewllian in the case of $\alpha_{e}$ $<0$, as seen in Figs. 4(a) and 4(b). On the other hand, the electron distribution function reduces to the state of few trapped electrons in the case of $\alpha_{e}>1$, as seen in Figs. 4(c) and 4(d).

\section{ION AND ELECTRON DENSITIES}

\section{A. Densities at the plug}

We assume that the charge neutrality conditions holds around the plug region, thus, the electrostatic potential $\phi$ around there is determined by the charge neutrality $n_{i}=n_{e}$. Requiring that $B_{p} \leqslant B_{i}$ and $\phi_{p} \geqslant \phi_{i}$, the ion density is obtained by integrating the distribution functions (1)-(6),

$$
\begin{aligned}
n_{i}= & n_{i c}\left[\exp \left\{-\Phi_{p}\right\}-\frac{\alpha_{i}\left(R_{i p}-1\right)}{\alpha_{i} R_{i p}-1}\left(\frac{R_{i p}-1}{R_{i p}}\right)^{1 / 2}\right. \\
& \left.\times \exp \left\{-\frac{R_{i p} \Phi_{p}}{R_{i p}-1}\right\}\right]+\frac{n_{i p}}{2}\left[\left(1-\alpha_{p}\right)^{1 / 2}\right. \\
& -\left(1-\alpha_{p}\right)^{1 / 2} \exp \left\{-\frac{\mu_{*} B_{p}}{T_{i}}\right\}-1-\frac{\left(1-\alpha_{p}\right)^{3 / 2}}{\alpha_{p} R_{m p}-1} \\
& \times \exp \left\{-\frac{\mu_{*} B_{p}}{T_{i}}\right\}+\frac{\alpha_{p}\left(R_{m p}-1\right)}{\alpha_{p} R_{m p}-1}\left(\frac{R_{m p}-1}{R_{m p}}\right)^{1 / 2} \\
& \left.\times \exp \left\{-\frac{\mu_{*} B_{p}}{T_{i}}\right\}\right] .
\end{aligned}
$$

Here $\Phi_{p} \equiv e\left[\phi_{p}-\phi_{i}\right] / T_{i}$ and $R_{i p} \equiv B_{i} / B_{p}, R_{m p} \equiv B_{m} / B_{p}$; $\operatorname{erf}\{\}$ and $\operatorname{erfc}\{\}$ are the error and complementary error functions defined by $\operatorname{erf}\{x\} \equiv 2 \int_{0}^{x} \exp \left\{-t^{2}\right\} d t / \sqrt{\pi}$, and $\operatorname{erfc}\{x\} \equiv 2$ $\times \int_{x}^{\infty} \exp \left\{-t^{2}\right\} d t / \sqrt{\pi}$, respectively. If $\alpha_{p}=0$, namely, if the distribution function of ionized ions is the same as that of coming back ions, which is obtained under the assumption that all ions passing over the plug and outer mirror throat come back to the central cell, the term proportional to $n_{i p}$ becomes zero in Eq. (15).

The electron density is given by Eqs. (13) and (14),

$n_{e}=\left\{\begin{array}{l}n_{e b}\left[\exp \left\{\Phi_{e}\right\} \operatorname{erfc}\left\{\sqrt{\Phi_{e}}\right\}+\frac{\alpha_{e}\left(R_{p b}-1\right)^{3 / 2}}{R_{p b}-\alpha_{e}} \operatorname{Ds}\left\{\left(\frac{\Phi_{e}}{R_{p b}-1}\right)^{1 / 2}\right\}-\frac{\left(\alpha_{e}-1\right)^{3 / 2} R_{p b}}{R_{p b}-\alpha_{e}} \operatorname{Ds}\left\{\left(\frac{\Phi_{e}}{\alpha_{e}-1}\right)^{1 / 2}\right\}\right] \\ n_{e b}\left[\exp \left\{\Phi_{e}\right\} \operatorname{erfc}\left\{\sqrt{\Phi_{e}}\right\}+\frac{\alpha_{e}\left(R_{p b}-1\right)^{3 / 2}}{R_{p b}-\alpha_{e}} \operatorname{Ds}\left\{\left(\frac{\Phi_{e}}{R_{p b}-1}\right)^{1 / 2}\right\}\right. \\ \left.\quad+\frac{\left(1-\alpha_{e}\right)^{3 / 2} R_{p b}}{R_{p b}-\alpha_{e}} \exp \left\{\frac{\Phi_{e}}{1-\alpha_{e}}\right\} \operatorname{erf}\left\{\left(\frac{\Phi_{e}}{1-\alpha_{e}}\right)^{1 / 2}\right\}\right] \quad \text { for } \quad \alpha_{e} \geqslant 1,\end{array}\right.$ 

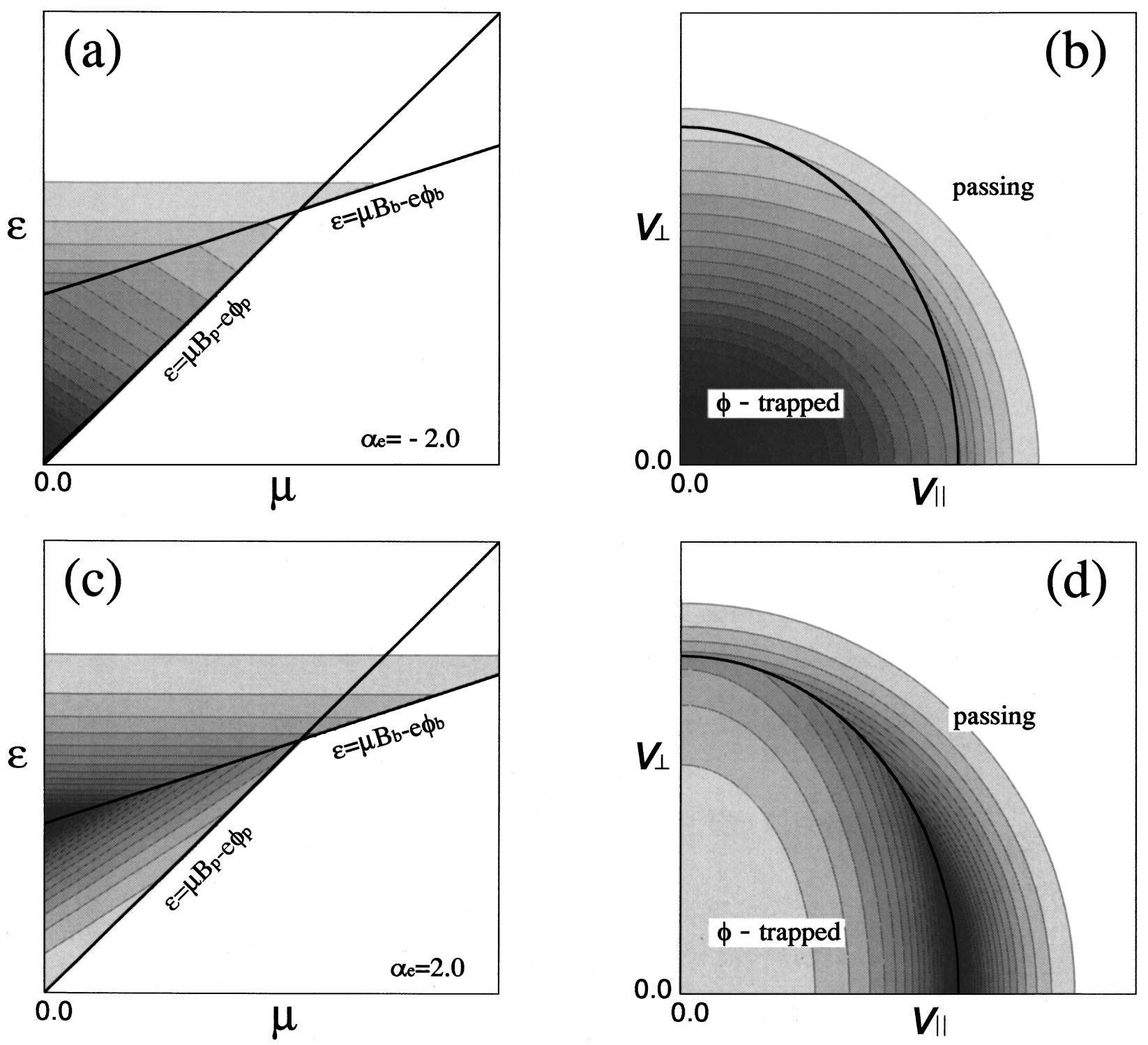

FIG. 4. The contour plot of the electron distribution function. In the figure, the parameters are $B_{p} / B_{b}=3.0, e\left(\phi_{p}-\phi_{b}\right) / T_{e}=3.0$, (a) and (c) are the velocity space described by $\epsilon$ and $\mu$, and (b) and (d) are the distribution function at the plug $z=z_{p}$, which corresponds to (a) and (c), respectively. The solid lines describe the separatrixes and the solid lines in (b) and (d) are the line $\epsilon=\mu B_{b}-e \phi_{b}$ in (a) and (c), respectively.

where $\Phi_{e} \equiv e\left[\phi_{p}-\phi_{b}\right] / T_{e}$ and $R_{p b} \equiv B_{p} / B_{b}$. The function $\operatorname{Ds}\{\}$ is defined as $\operatorname{Ds}\{x\} \equiv 2 \exp$ $\left\{-x^{2}\right\} \int_{0}^{x} \exp \left\{t^{2}\right\} d t / \sqrt{\pi}$.

The charge neutrality condition $n_{i}=n_{e}$ gives the electrostatic potential as a function of $B$, i.e., $\phi=\phi(B)$. The plug potential $\phi_{p}$ should satisfy the conditions that $\phi_{p}>\phi_{i}$ and $d \phi\left(B_{p}\right) / d B_{p}=0$ because the plug is a position of the poten- tial maximum. Provided that the density is differentiable with $B$ and $\phi$, it is written as $d \phi\left(B_{p}\right) / d B_{p}=-\left[\partial n_{i} / \partial B_{p}\right.$ $\left.-\partial n_{e} / \partial B_{p}\right] /\left[\partial n_{i} / \partial \phi_{p}-\partial n_{e} / \partial \phi_{p}\right]$. Therefore, the plug potential should satisfy both conditions that $n_{i}\left(B_{p}, \phi_{p}\right)$ $=n_{e}\left(B_{p}, \phi_{p}\right)$ and $\partial n_{i}\left(B_{p}, \phi_{p}\right) / \partial B_{p}=\partial n_{e}\left(B_{p}, \phi_{p}\right) / \partial B_{p}$. The explicit form of the derivative of each density with respect of $B$ at $B=B_{p}$ is given by

$$
\begin{aligned}
\frac{\partial n_{i}}{\partial B_{p}}= & \frac{n_{i c}}{B_{i}} \exp \left\{-\frac{R_{i p} \Phi_{p}}{R_{i p}-1}\right\}\left[\frac{\alpha_{i}\left(R_{i p}-1\right)}{\alpha_{i} R_{i p}-1}\left\{\frac{1}{2}\left(\frac{R_{i p}}{R_{i p}-1}\right)^{1 / 2}+\left(\frac{R_{i p}}{R_{i p}-1}\right)^{3 / 2} \Phi_{p}\right\}+\frac{\alpha_{i}\left(\alpha_{i}-1\right) R_{i p}^{2}}{\left(\alpha_{i} R_{i p}-1\right)^{2}}\left(\frac{R_{i p}-1}{R_{i p}}\right)^{1 / 2}\right] \\
& +\frac{n_{i p}}{2 B_{p}} \frac{\alpha_{p}}{\left(1-\alpha_{p}\right)^{1 / 2}} \exp \left\{-\frac{\mu_{*} B_{p}}{T_{i}}\right\}\left[1-\exp \left\{\frac{\mu_{*} B_{p}}{T_{i}}\right\}-\frac{\left(1-\alpha_{p}\right)^{2} R_{m p}}{\left(1-\alpha_{p} R_{m p}\right)^{2}}\right. \\
& \left.+\frac{\left(1-\alpha_{p}\right)^{1 / 2}\left\{1+\left(2-3 \alpha_{p}\right) R_{m p}\right\}}{2\left(1-\alpha_{p} R_{m p}\right)^{2}}\left(\frac{R_{m p}-1}{R_{m p}}\right)^{1 / 2}-\frac{\left(R_{m p}-1\right) \mu_{*} B_{p}}{\left(1-\alpha_{p} R_{m p}\right) T_{i}}+\frac{\left(1-\alpha_{p}\right)^{1 / 2} R_{m p} \mu_{*} B_{p}}{\left(1-\alpha_{p} R_{m p}\right) T_{i}}\left(\frac{R_{m p}-1}{R_{m p}}\right)^{1 / 2}\right],
\end{aligned}
$$




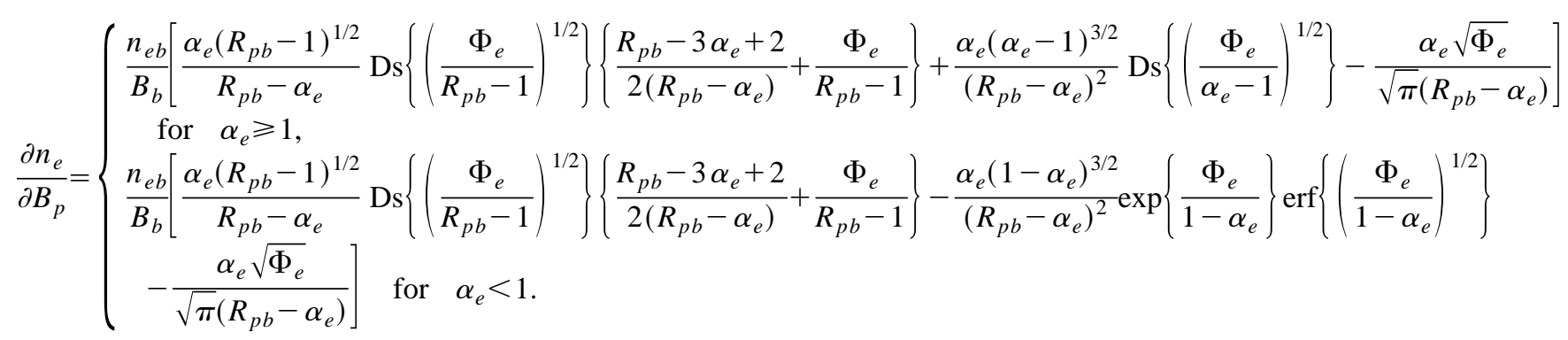

As seen in Eq. (17), the magnitude of $\partial n_{i} / \partial B_{p}$ grows infinitely large as $\alpha_{p}$ approaches unity. Thus, the plug potential does not form if none of the ions are produced by the ionization around the plug region. For the case of $\alpha_{p}=0$, the ion distribution function is symmetric around the line of $v_{\|}=0$, as seen in Eqs. (1)-(6). Throughout this manuscript, we assume $T_{i}=T_{e}$.

The procedure to find the solution of $\phi_{p}$ and $B_{p}$ is the following: At first, we find the solution $\phi^{\alpha}=\phi^{\alpha}\left(B_{p}\right)$, satisfying the equation $n_{i}\left(\phi^{\alpha}, B_{p}\right)=n_{e}\left(\phi^{\alpha}, B_{p}\right)$. At the same time, the solution $\phi^{\beta}=\phi^{\beta}\left(B_{p}\right)$ is determined by solving the equation $\partial n_{i}\left(\phi^{\beta}, B_{p}\right) / \partial B_{p}=\partial n_{e}\left(\phi^{\beta}, B_{p}\right) / \partial B_{p}$. The plug potential is obtained if the two solutions $\phi^{\alpha}\left(B_{p}\right)$ and $\phi^{\beta}\left(B_{p}\right)$ are equal.

Figure 5 displays an example of $\phi^{\alpha}=\phi^{\alpha}\left(B_{p}\right)$ and $\phi^{\beta}$ $=\phi^{\beta}\left(B_{p}\right)$. One can see that there is one intersection of the two solutions in both Figs. 5(a) and 5(b). It is seen that there is a position of the potential maximum between $B / B_{b}=1$ and $B / B_{b}=6\left(=B_{m} / B_{b}\right)$. Figure 5 demonstrates that the plug potential is created only by ions coming from the central cell, ions trapped in the thermal barrier region, and ions born around the plug region but without resorting to highenergy sloshing ions, where the detailed analysis is given in the paper. ${ }^{20}$

\section{B. Ion density in the region $z>z_{p}$}

It is demonstrated in Sec. III A that the plug potential is created between $z=z_{b}$ and $z=z_{m}$ within the present model. The ion density at the plug is determined by using the ion distribution function, which is defined in the range $z_{b} \leqslant z$ $\leqslant z_{p}$, so that the electrostatic potential is continuous from $z=z_{b}$ to $z=z_{p}$. The next problem, therefore, is to find the condition in which the potential is continuous in the region $z \geqslant z_{p}$.

The ion density is obtained by integrating the ion distribution functions (7)-(12) in the velocity space, the explicit form of which is too lengthy to be written down in this manuscript.

The conditions in which $n_{i}(B, \phi)=n_{e}(B, \phi)$ and $\partial n_{i}(B, \phi) / \partial B=\partial n_{e}(B, \phi) / \partial B$ at $z=z_{p}$ are required for the plug potential formation. The magnitude and location of the plug potential are determined by using the ion and electron densities in Sec. III A, where the ion distribution function defined in the region $z \leqslant z_{p}$ is used. In order that the electrostatic potential in the region $z \leqslant z_{p}$ connects smoothly with that in the region $z>z_{p}$, the differential $\partial n_{i}(B, \phi) / \partial B$ calculated by the ion distribution function defined in $z>z_{p}$ is required to be the same as the differential in Eq. (17). The straightforward but tedious calculation verifies that the differential $\partial n_{i}(B, \phi) / \partial B$, by using the ion distribution functions in Eqs. (7) and (8)-(12), coincides with the differential (17) as long as the condition $d \phi(B) / d B=0$ is satisfied at $z$ $=z_{p}$.

The explicit form of the electron density $n_{e}(\phi, B)$ in $z$ $\geqslant z_{p}$ is the same as that in the region $z \leqslant z_{p}$, which is given by Eq. (16) by replacing $B_{p}$ and $\phi_{p}$ (i.e., $R_{p b}$ and $\Phi_{e}$ ) with $B$ and $\phi$ (i.e., $R_{b}$ and $\Phi_{e z}$ ), respectively:

$$
n_{e}=\left\{\begin{array}{l}
n_{e b}\left[\exp \left\{\Phi_{e z}\right\} \operatorname{erfc}\left\{\sqrt{\Phi_{e z}}\right\}+\frac{\alpha_{e}\left(R_{b}-1\right)^{3 / 2}}{R_{b}-\alpha_{e}} \operatorname{Ds}\left\{\left(\frac{\Phi_{e z}}{R_{b}-1}\right)^{1 / 2}\right\}-\frac{\left(\alpha_{e}-1\right)^{3 / 2} R_{b}}{R_{b}-\alpha_{e}} \operatorname{Ds}\left\{\left(\frac{\Phi_{e z}}{\alpha_{e}-1}\right)^{1 / 2}\right\}\right] \\
\quad \text { for } \alpha_{e} \geqslant 1, \\
n_{e b}\left[\exp \left\{\Phi_{e z}\right\} \operatorname{erfc}\left\{\sqrt{\Phi_{e z}}\right\}+\frac{\alpha_{e}\left(R_{b}-1\right)^{3 / 2}}{R_{b}-\alpha_{e}} \operatorname{Ds}\left\{\left(\frac{\Phi_{e z}}{R_{b}-1}\right)^{1 / 2}\right\}+\frac{\left(1-\alpha_{e}\right)^{3 / 2} R_{b}}{R_{b}-\alpha_{e}} \exp \left\{\frac{\Phi_{e z}}{1-\alpha_{e}}\right\} \operatorname{erf}\left\{\left(\frac{\Phi_{e z}}{1-\alpha_{e}}\right)^{1 / 2}\right\}\right] \\
\quad \text { for } \alpha_{e}<1,
\end{array}\right.
$$

where $\Phi_{e z} \equiv e\left[\phi-\phi_{b}\right] / T_{e}$ and $R_{b} \equiv B / B_{b}$.

\section{THE ELECTROSTATIC POTENTIAL}

\section{A. The electrostatic potential around the plug}

In this section, we show that the plug potential is formed for various parameters of $\alpha_{i}, \alpha_{e}$, and $\alpha_{p}$. As is displayed in Sec. III A the solution that satisfies the conditions $n_{i}(\phi, B)$ $=n_{e}(\phi, B)$ and $\partial n_{i}(\phi, B) / \partial B=\partial n_{e}(\phi, B) / \partial B$ can exist between $z=z_{b}$ and $z=z_{m}$.

At first, the results with $\alpha_{p}=1$ are shown, where the ion distribution function does not have a loss cone distribution. 

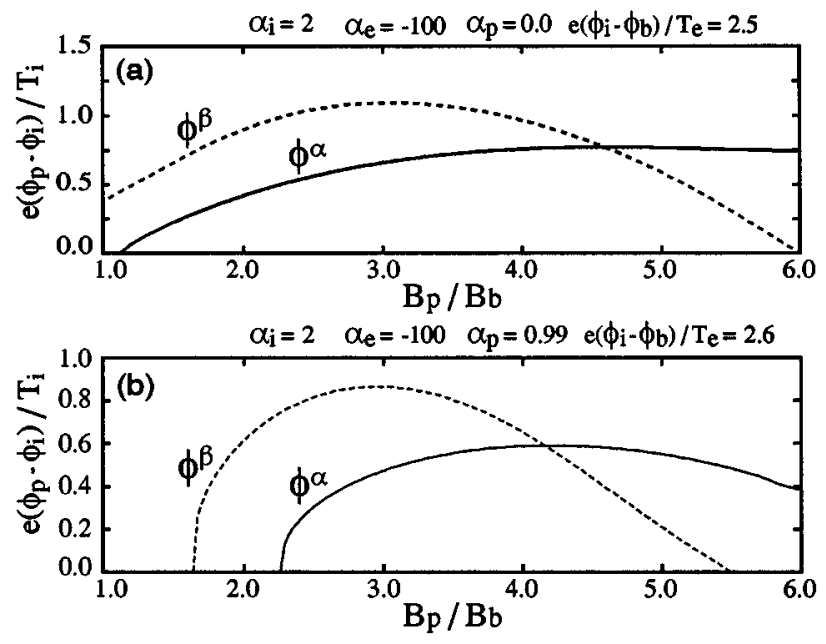

FIG. 5. The solutions $\phi^{a}$ and $\phi^{\beta}$ as a function of $B_{p} / B_{b}$.

This case does not require the information on the outer mirror throat, so that this is the simplest case for the plug potential formation.

Figure 6(a) displays the plug potential (ion-confining potential) $e\left(\phi_{p}-\phi_{i}\right) / T_{i}$ as a function of the thermal barrier potential depth $e\left(\phi_{i}-\phi_{b}\right) / T_{e}$. One can see the plug potential can form in a wide range of thermal barrier depths and $\alpha_{e}$. In the case of $\alpha_{e}>1$, the plug potential is not created because the temperature of the electrons trapped in the plug potential is lower than that of the passing electrons. That is, the plug potential formation requires electron heating around the plug region by an externally injected microwave. The higher plug potential is created with deeper thermal barrier potential depth. On the other hand, the location of the plug potential moves to the position of lower magnitude of the magnetic field with a deeper thermal barrier. It is found that the electron distribution function influences both the magnitude and location of the plug potential.

Figure 6(b) displays the plug potential as a function of thermal barrier potential depth as in Fig. 6(a). In Fig. 6(b) the coefficient $\alpha_{e}$ is fixed. It is seen that the plug potential can be formed for a wide range of thermal barrier depths and $\alpha_{i}$. The remarkable feature in Fig. 6(b) is that the height of the plug potential does not depend on $\alpha_{i}$ so much, but it becomes larger with deeper thermal barrier depth.

Through Figs. 6(a) and 6(b), the location of the plug changes with the thermal barrier depth even if the coefficients $\alpha_{i}$ and $\alpha_{e}$ are fixed. The ion and electron distributions change with the thermal barrier potential depth because the separatrix distinguishing the trapped and passing particles changes with thermal barrier depth.

The following results are the cases in which the ion distribution function has a loss cone. The electrostatic potential at $z=z_{m}$ is given in advance through the quantity $\mu_{*}$ in the plug potential formation.

Figure 7(a) displays the case of $\mu_{*} B_{p} / T_{i}=0.1$. The constant $\alpha_{p}$ represents the distribution function in the loss cone. With the assumption that the distribution function decreases in the loss cone region from the loss cone boundary, the magnitude of $\alpha_{p}$ takes a value between 0 and 1 . The plug
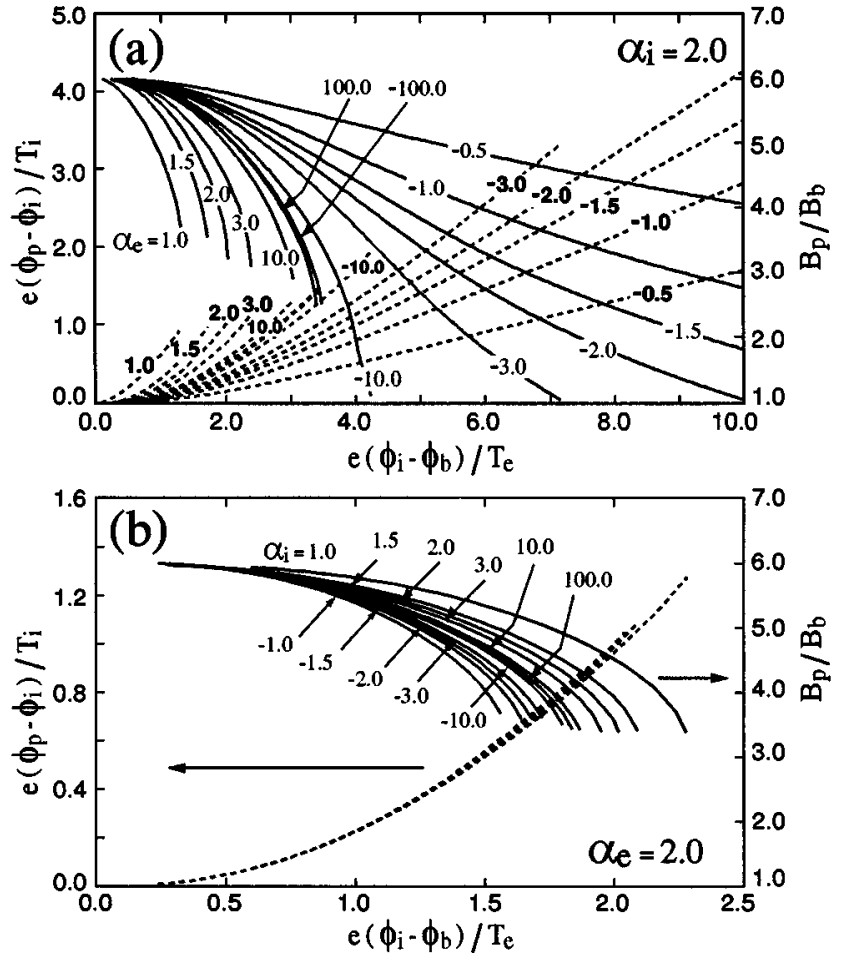

FIG. 6. The magnitude of the plug potential as a function of thermal barrier depth in the case of $\alpha_{p}=1$. Solid lines represent the location of plug, i.e., $B_{p} / B_{b}$, and dashed lines are the magnitude of the plug potential; (a) is the case of $\alpha_{i}=2.0$ and (b) is $\alpha_{e}=2.0$.

potential is found to exist in a wide range of $\alpha_{p}$, where the $\alpha_{p}$ have an effect on the location of the plug potential as well as its magnitude. As mentioned in Sec. III A, the leading term of $\partial n_{i}\left(\phi_{p}, B_{p}\right) / \partial B_{p}$ in Eq. (17) is proportional to $n_{i p} \alpha_{p} /\left[B_{p}\left(1-\alpha_{p}\right)^{1 / 2}\right]$, so that as $\alpha_{p}$ approaches unity, the differential $n_{i p} / B_{p}$ becomes larger in order to satisfy the relation $\partial n_{i} / \partial B_{p}=\partial n_{e} / \partial B_{p}$. This is the reason why the ratio of $B_{p}$ to $B_{b}$ is smaller with larger $\alpha_{p}$ at the same thermal barrier depth in Fig. 7(a).

The results for various $\mu_{*} B_{p} / T_{i}$ are shown in Figs. 7(b) and $7(\mathrm{c})$. As the definition, the value of $\mu_{*}$ is positive definite, so that the positive cases are shown in Fig. 7. The solution is not found in the case of $\mu_{*} B_{p} / T_{i}$ being larger than 1.2 for $\alpha_{p}=0.9$, while there is no solution in the region where $\mu_{*} B_{p} / T_{i}$ is larger than 0.4 for $\alpha_{p}=0.99$. The location of the plug potential is much more influenced by the $\mu_{*} B_{p} / T_{i}$ than the magnitude of the plug potential. The region where the plug potential exists in Fig. 7(b) almost coincides with that in Fig. 7(c).

In summary of this section, the plug potential can form easily within the present model. The height of the plug potential increases with the thermal barrier depth. The location of the plug potential also changes with the thermal barrier depth, where the location moves to a lower magnetic field with a deeper thermal barrier depth. The region where the plug potential exists does not depend on $\alpha_{p}$ as much.

\section{B. Potential profile beyond the plug}

The plug potential is obtained by using the ion distribution function defined in the region $z \leqslant z_{p}$, where the condi- 

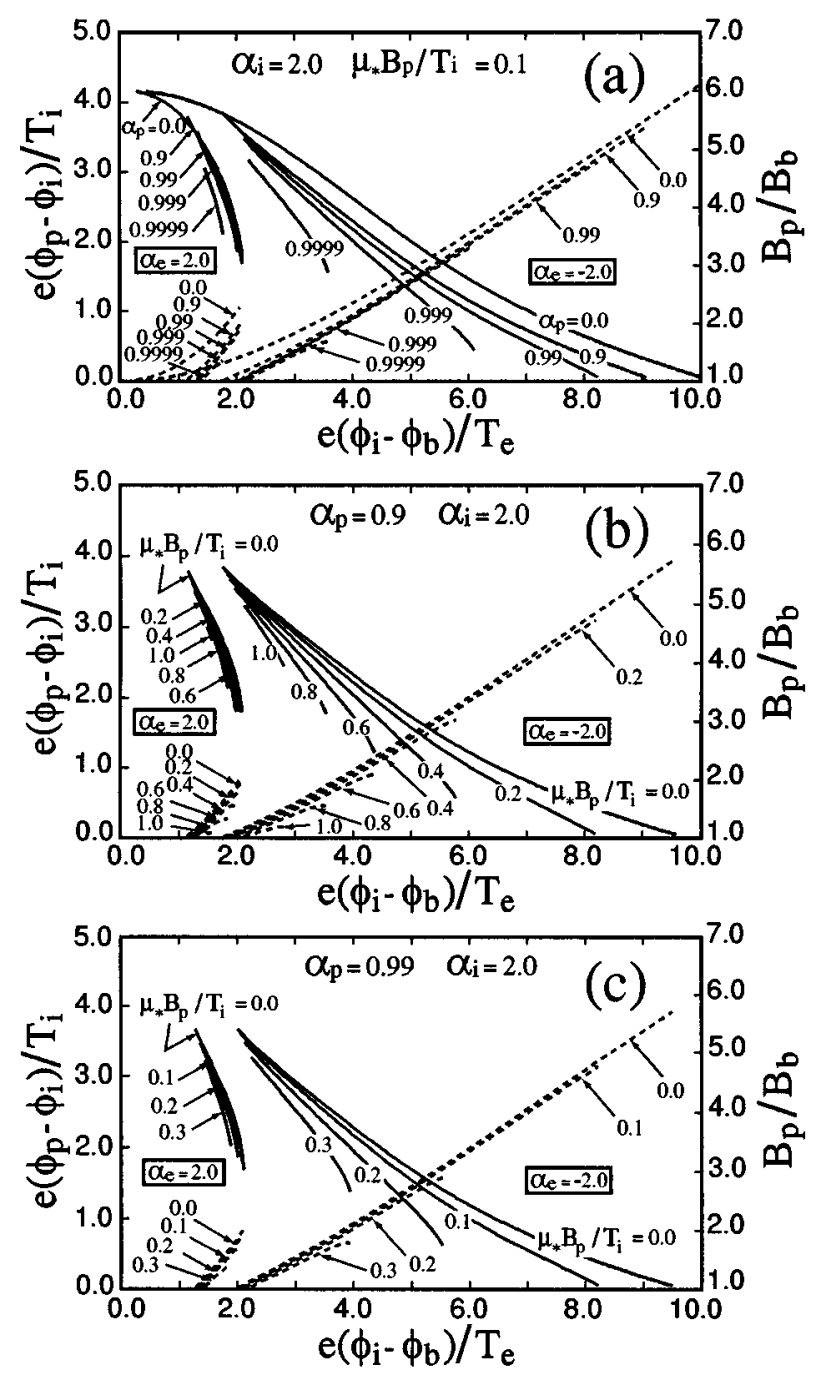

FIG. 7. The magnitude of the plug potential as a function of thermal barrier depth in the case of $\alpha_{p} \neq 1$. Solid lines represent the location of the plug, i.e., $B_{p} / B_{b}$, and dashed lines are the magnitude of the plug potential; (a) is the case of $a_{i}=2.0$ and $\mu_{*} B_{p} / T_{i}=0.1$, (b) is the case of $\alpha_{p}=0.9$ and $\alpha_{i}$ $=2.0$, and (c) is the case of $\alpha_{p}=0.99$ and $\alpha_{i}=2.0$.

tion $d \phi\left(B_{p}\right) / d B_{p}=0$ is derived from the potential solved in the region $z \leqslant z_{p}$. The potential just inside the plug, i.e., $z$ $\leqslant z_{p}$, therefore, is continuous, that is, there is no potential jump. This is because there exists a population of ions produced around the plug and going toward the central cell, i.e., $\alpha_{p} \neq 1$, and a population of passing ions flowing from the central cell.

There is a possibility of sheath potential formation in the region $z>z_{p}$. In this section, therefore, the condition under which the potential connects continuously across the plug is made clear.

It is well known that the sheath potential generates in such a case, so that the ions with zero drift velocity are accelerated by the electrostatic potential and then are absorbed at an end wall. The situation at the plug is just the same as the sheath potential formation in the case of $\alpha_{q}$ $=1$. The only different point from the sheath formation is that there exists a population of ions passing through the

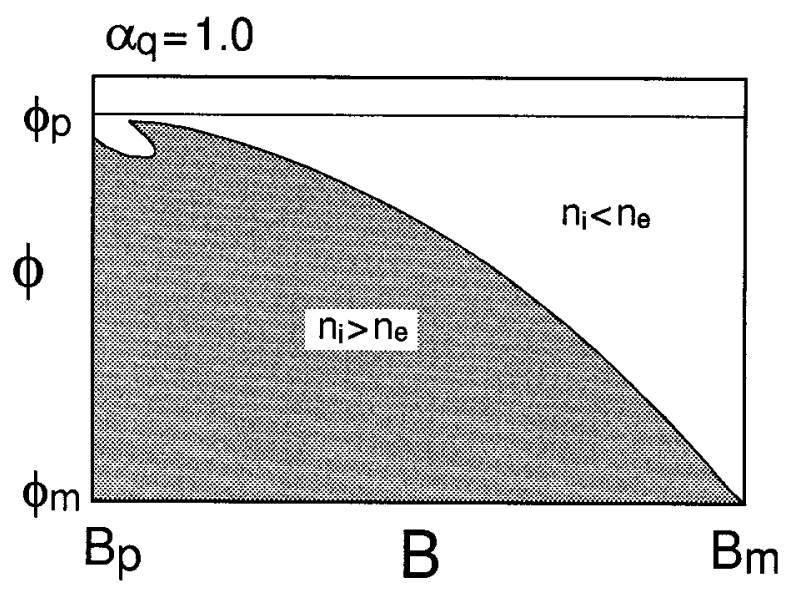

FIG. 8. Contour plots of $n_{i}-n_{e}$. The dark colored region is $n_{i}>n_{e}$ and the white region is $n_{i}<n_{e}$.

plug and then being reflected by the magnetic field at the outer mirror throat.

Figure 8 shows an example of the potential calculated by the charge neutrality condition by using electron density (18) and the ion density, the explicit form of which is not written in the manuscript. Here, to display the quasineutral profile, we plot the contour surface of the zero-level of $n_{i}-n_{e}$ as a function of $B / B_{p}$ and $e\left(\phi-\phi_{p}\right) / T_{e}$. This procedure ensures that we will find all possible roots of $n_{i}=n_{e}$. The parameters used in Fig. 8 and $B_{i} / B_{b}=6, e\left(\phi_{i}-\phi_{b}\right) / T_{e}=2.3, \alpha_{i}=-2$, $\alpha_{e}=-100, \alpha_{x}=0.9, \alpha_{q}=1$, and $\mu_{*} B_{P} / T_{i}=0.37$. The location and magnitude of the plug potential are determined by solving $n_{i}\left(\phi_{p}, B_{p}\right)=n_{e}\left(\phi_{p} . B_{p}\right) \quad$ and $\quad \partial n_{i}\left(\phi_{p}, B_{p}\right) / \partial B_{p}$ $=\partial n_{e}\left(\phi_{p}, B_{p}\right) / \partial B_{p}$. The resultant plug exists at $B_{p} / B_{b}=3.3, \quad e\left(\phi_{p}-\phi_{i}\right) / T_{i}=0.31, \quad$ and $\quad e\left(\phi_{m}-\phi_{i}\right) / T_{i}$ $=0.012$.

The dark painted region is where $n_{i}-n_{e}$ is positive, while $n_{i}-n_{e}$ is negative in the white region. The boundary, therefore, represents the possible root of $n_{i}-n_{e}=0$ in Fig. 8. It is seen that the potential jump is observed at $z=z_{p}$. The actual electrostatic potential is determined, in general, with the help of the Poisson equation $\Delta \phi=-4 \pi e\left(n_{i}-n_{e}\right)$. The solution for $\phi$ obtained from the Poisson equation is well approximated by the one obtained from the quasineutral equation $n_{i}=n_{e}$, where the two solutions differs by $O\left(\lambda_{D}^{2} / L^{2}\right)$, and the solution to the Poisson equation satisfies the charge neutrality to the same order. Here, $\lambda_{D}$ and $L$ are the Debye length and the scale length for potential variation, respectively.

When the potential is smoothly varying with $L \gg \lambda_{D}$, the assumption of quasineutrality provides a good approximation to the solution of the Poisson equation. The jump of the potential, however, shows the breaking of the charge neutrality condition at $z=z_{p}$, so that the Poisson equation, instead of quasineutrality, should be applied there. The sheath potential, therefore, is formed at $z=z_{p}$.

Another remarkable feature in Fig. 8 is that $\phi\left(B / B_{p}\right)$ has a multivalued solution at the position finely different from $B / B_{p}=1$, so that the full Poisson equation treatment is required to establish the true potential there. The solutions of 

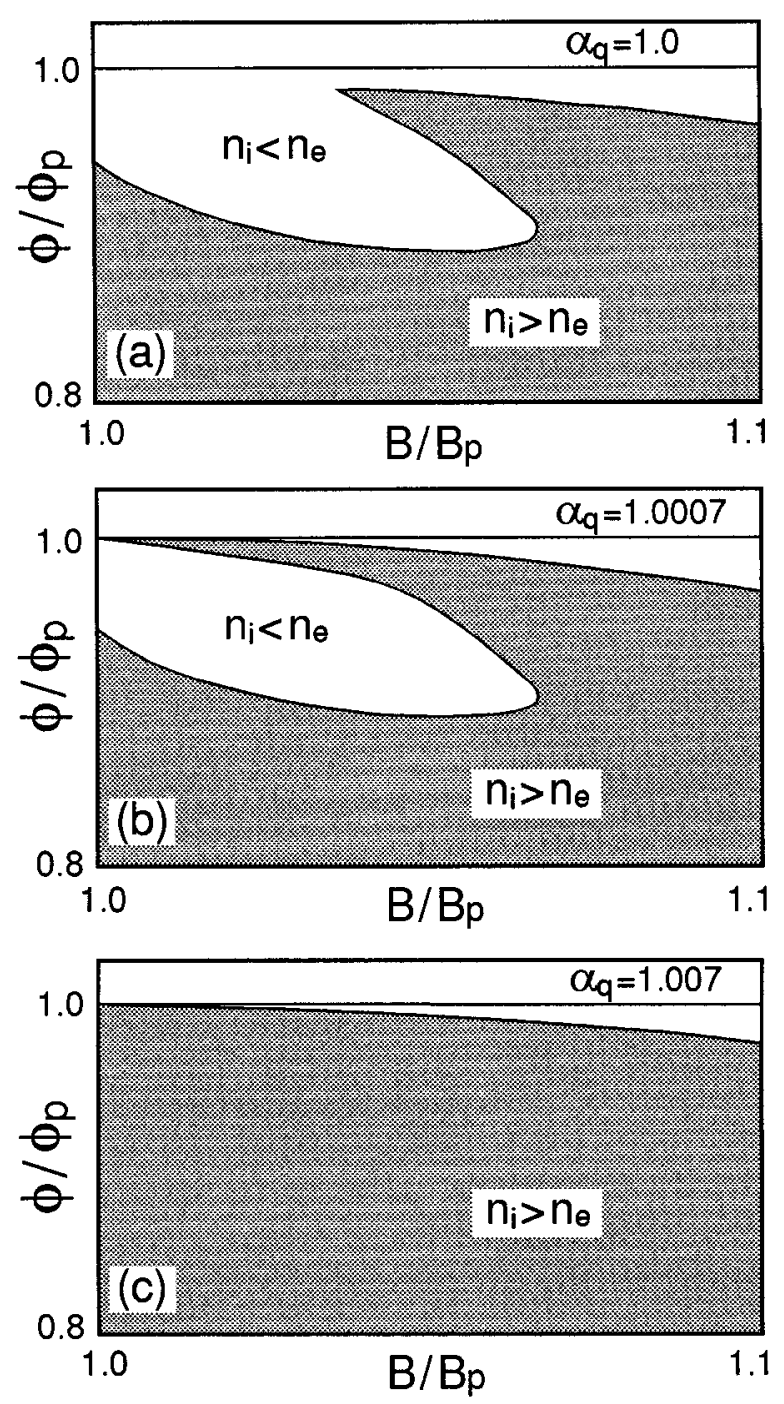

FIG. 9. Contour plots of $n_{i}-n_{e}$. The dark colored region is $n_{i}>n_{e}$ and the white region is $n_{i}<n_{e}$. (a), (b), and (c) are the cases of $\alpha_{q}=1.0,1.0007$, and 1.007 , respectively.

the Poisson equation vary with the boundary condition, that is, whether starting from $z=z_{p}$ or from the end wall.

Whichever boundary condition is chosen, there is a minimum region of potential between $z=z_{p}$ and the end wall

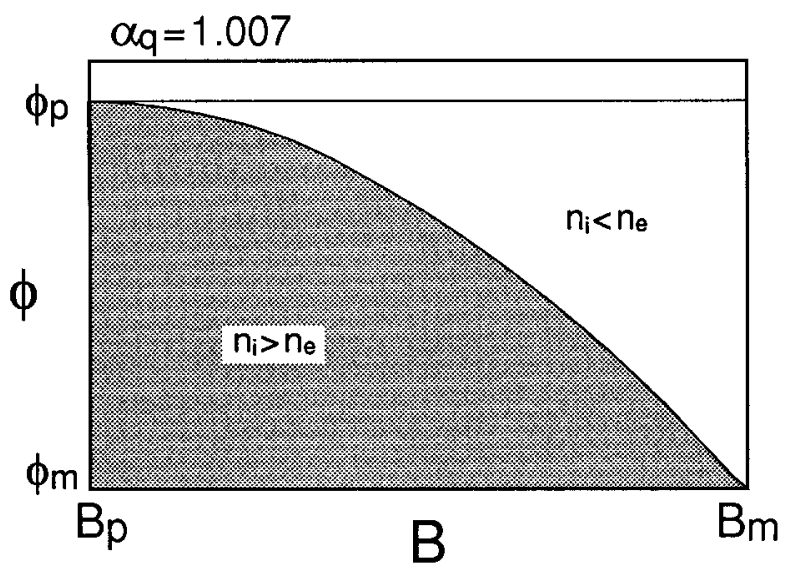

FIG. 10. Contour plots of $n_{i}-n_{e}$ in the case of $\alpha_{q}=1.007$. The dark colored region is $n_{i}>n_{e}$ and the white region is $n_{i}<n_{e}$.
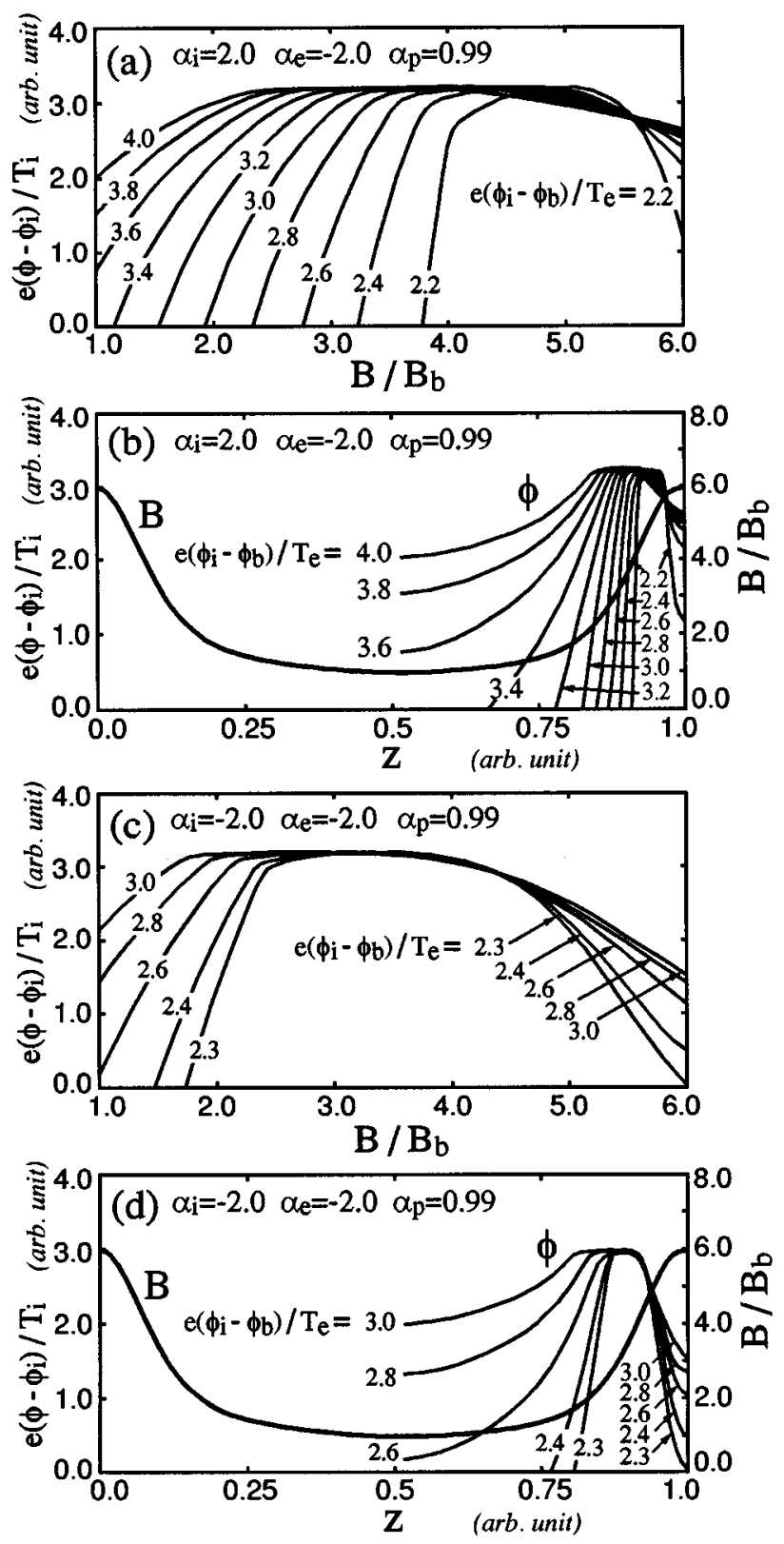

FIG. 11. Potential profiles for varous thermal barrier potential depths: (a) and (c) are a function of $B / B_{b}$, and (b) and (d) are plotted as a function of $z$. Here, the parameters used are that of $\alpha_{e}=-2.0, \alpha_{p}=0.99, \alpha_{x}=0.9, \alpha_{p}$ $=1.01$, and $\mu_{*} B_{p} / T_{i}=0.2$ in all figures. Especially (a) and (b) are the case of $\alpha_{i}=2.0$, and (c) and (d) are $\alpha_{i}=-2.0$. The magnetic-field profile used in (b) and (d) is that in the end mirror cell of the GAMMA 10 tandem mirror.

in Fig. 8, where ions are trapped in the potential well by Coulomb pitch angle scattering, leading to another state of electrostatic potential. To determine the resultant potential profile, the ion distribution function trapped in the potential well must be determined, which is beyond the scope of this manuscript. Even if the distribution function of ions trapped in the potential well is determined and the potential profile is recalculated by using the distribution function, the sheath potential will remain at $z=z_{p}$.

The cases of $\alpha_{q} \neq 1$ are shown in Fig. 9. When $\alpha_{q}=1.0007$, there is no potential jump around $z=z_{p}$. Here, the change of $\alpha_{q}$ slightly away from 1.0 takes into account the ions that are born around the plug and escape toward the 
outer mirror throat (i.e., ions indicated by orbit (8) in Fig. 2). Although the potential has the multivalued solution in the neighborhood of $z=z_{p}$ in Fig. 9(b), the smooth potential profile is obtained. The state, however, is not stable. By a slight variation of potential height at $z=z_{p}$, the topology of the potential profile shifts to that in Fig. 9(a), so that the small oscillation of the potential at $z=z_{p}$ leads to a large oscillation around $z=z_{p}$. In the case of $\alpha_{q}=1.007$, the potential has a single valued solution around $z=z_{p}$, as shown in Fig. 9(c).

The potential profile from $z=z_{p}$ to $z=z_{m}$ is plotted in Fig. 10 for the case of $\alpha_{q}=1.007$. It is found that the singlevalued solution of the potential, being maximum at $z=z_{p}$ and decreasing toward $z=z_{m}$, exists. The formation of the multivalued solution, such as in Fig. 9(a), is due to the existence of the outer mirror throat. If there is not an outer mirror throat, i.e., there is not a gradient of the magnitude of the magnetic field, the present problem is the same as a normal problem of the sheath potential formation. Figure 10 indicates that there is a potential profile, which is maximum at $z=z_{p}$ with $d \phi\left(B_{p}\right) / d B_{p}=0$, and decreases toward the outer mirror throat within the present model.

The potential is found to be continuous across the plug [as in Fig. 9(b)] when $\alpha_{q}$ is larger than 1.0002-1.0012, depending on the parameters of $\alpha_{i}, \alpha_{e}$, and $\mu_{*} B_{p} / T_{i}$.

\section{Plug potential profile}

The magnitude and location of the plug potential are investigated in Sec. IV A and the potential profile from the plug to the outer mirror throat is demonstrated in Sec. IV B. So, the remaining problem is the potential profile around the plug, which is shown in this section. The potential profile is obtained by the charge neutrality condition of the ion and electron densities.

The ion density in the regions $z \leqslant z_{p}$ and $\phi_{p} \geqslant \phi \geqslant \phi_{i}$ is given by integrating the distribution functions (1)-(6) in the appropriate velocity space, though the explicit form of the ion density is not written down in this manuscript because the form is too lengthy.
At first, the magnitude and position of the plug potential are determined by the method described in Sec. III A. And, then the potential is solved by the ion and electron charge neutrality condition.

Figure 11 shows the various potential profiles determined by the charge neutrality condition. As can be seen in Fig. 11, the continuous potential profile, which has its maximum at a point midway between $z=z_{m}$ and $z=z_{b}$, is obtained for various thermal barrier depths. The maximum position of the potential profile moves to the lower magneticfield side as the thermal barrier potential depth is deeper in Fig. 11(a), which is consistent with the results in Sec. IV A. The width of the potential becomes larger with the deeper thermal barrier depth.

The calculated potentials at $z=z_{b}$ are larger than $\phi_{i}$ in the case of $e\left(\phi_{i}-\phi_{b}\right) / T_{e} \geqslant 3.6$ in Figs. 11(a) and 11(b), and larger than $\phi_{i}$ in $e\left(\phi_{i}-\phi_{b}\right) T_{e} \geqslant 2.6$ in Figs. 11(c) and 11(d). There is a population of magnetically trapped hot electrons around $z=z_{b}$ in an actual tandem mirror, which is neglected in this manuscript. The hot electron population should be taken into account for the potential in the neighborhood of $z=z_{b}$, which will make the magnitude of the potential lower than $\phi_{i}$ around $z=z_{b}$. The important point in Fig. 11 is that the potential has a maximum between $z=z_{b}$ and $z=z_{m}$.

\section{DISCUSSION}

The formation of a continuous plug potential requires the ion distribution function to be characterized by $\alpha_{p}$ in Eqs. (4) and (5) and by $\alpha_{q}$ in Eq. (12). Those ions are assumed to be supplied by the ionization process around the plug and to escape to the central cell and/or end wall. So, we estimate the magnitudes of $\alpha_{p}$ and $\alpha_{q}$ in the following.

We consider the ion distribution function $F_{i}$. The distribution function $v_{\|}>0$ (defined by $F_{i}^{+}$and $\widetilde{F}_{i}^{+}$) is that escape to the end wall and the distribution function $v_{\|}<0$ (defined by $\widetilde{F}_{i}^{-}$) corresponds to those escaping to the central cell. That is,

$$
\begin{aligned}
& F_{i}^{+}=n_{i c}\left(\frac{m_{i}}{2 \pi T_{i}}\right)^{3 / 2} \exp \left\{-\frac{\epsilon-e \phi_{i}}{T_{i}}\right\}, \quad \text { for } \epsilon>\mu B_{m}+e \phi_{m} \text { and } \epsilon>\mu B_{p}+e \phi_{p}, \\
& \widetilde{F}_{i}^{+}=n_{i p}\left(\frac{m_{i}}{2 \pi T_{i}}\right)^{3 / 2} \exp \left\{-\frac{\epsilon-e \phi_{p}-\alpha_{q} \mu B_{p}}{\left(1-\alpha_{q}\right) T_{i}}\right\}, \quad \text { for } \epsilon>\mu B_{m}+e \phi_{m} \text { and } \epsilon \leqslant \mu B_{p}+e \phi_{p}, \\
& \widetilde{F}_{i}^{-}=n_{i p}\left(\frac{m_{i}}{2 \pi T_{i}}\right)^{3 / 2} \exp \left\{-\frac{\epsilon-e \phi_{p}-\alpha_{p} \mu B_{p}}{\left(1-\alpha_{p}\right) T_{i}}\right\}, \quad \text { for } \epsilon>\mu B_{p}+e \phi_{p} \text { and } \mu<\mu_{*} .
\end{aligned}
$$

Now, we calculate the particle fluxes $J_{i}^{+}, \widetilde{J}_{i}^{+}$, and $\widetilde{J}_{i}^{-}$at $z=z_{p}$, where 


$$
\begin{aligned}
& J_{i}^{+} \equiv \frac{2 \pi B_{p}}{m_{i}^{2}} \int_{0}^{\mu_{*}} d \mu \int_{\mu B_{p}+e \phi_{p}}^{\infty} d \epsilon n_{i c}\left(\frac{m_{i}}{2 \pi T_{i}}\right)^{3 / 2} \exp \left\{-\frac{\epsilon-e \phi_{i}}{T_{i}}\right\}+\frac{2 \pi B_{p}}{m_{i}^{2}} \int_{\mu_{*}}^{\infty} d \mu \int_{\mu B_{m}+e \phi_{m}}^{\infty} d \epsilon n_{i c}\left(\frac{m_{i}}{2 \pi T_{i}}\right)^{3 / 2} \\
& \times \exp \left\{-\frac{\epsilon-e \phi_{i}}{T_{i}}\right\} \\
& =n_{i c} \exp \left\{-\frac{e \phi_{p}-e \phi_{i}}{T_{i}}\right\} \sqrt{\frac{T_{i}}{2 \pi m_{i}}}\left[1-\frac{B_{m} / B_{p}-1}{B_{m} / B_{p}} \exp \left\{-\frac{\mu_{*} B_{p}}{T_{i}}\right\}\right] \\
& \widetilde{J}_{i}^{+} \equiv \frac{2 \pi B_{p}}{m_{i}^{2}} \int_{0}^{\mu_{*}} d \mu \int_{\mu B_{m}+e \phi_{m}}^{\mu B_{p}+e \phi_{p}} d \epsilon n_{i p}\left(\frac{m_{i}}{2 \pi T_{i}}\right)^{3 / 2} \exp \left\{-\frac{\epsilon-e \phi_{p}-\alpha_{q} \mu B_{p}}{\left(1-\alpha_{q}\right) T_{i}}\right\} \\
& =-n_{i p} \sqrt{\frac{T_{i}}{2 \pi m_{i}}}\left(1-\alpha_{q}\right)\left[1-\frac{\left(1-\alpha_{q}\right)}{B_{m} / B_{p}-\alpha_{q}} \exp \left\{-\frac{e \phi_{m}-e \phi_{p}}{\left(1-\alpha_{q}\right) T_{i}}\right\}-\frac{B_{m} / B_{p}-1}{B_{m} / B_{p}-\alpha_{q}} \exp \left\{-\frac{\mu_{*} B_{p}}{T_{i}}\right\}\right] \text {, } \\
& \widetilde{J}_{i}^{-} \equiv \frac{2 \pi B_{p}}{m_{i}^{2}} \int_{0}^{\mu_{*}} d \mu \int_{\mu B_{p}+e \phi_{p}}^{\infty} d \epsilon n_{i p}\left(\frac{m_{i}}{2 \pi T_{i}}\right)^{3 / 2} \exp \left\{-\frac{\epsilon-e \phi_{p}-\alpha_{p} \mu B_{p}}{\left(1-\alpha_{p}\right) T_{i}}\right\} \\
& +\frac{2 \pi B_{p}}{m_{i}^{2}} \int_{\mu_{*}}^{\infty} d \mu \int_{\mu B_{m}+e \phi_{m}}^{\infty} d \epsilon n_{i p}\left(\frac{m_{i}}{2 \pi T_{i}}\right)^{3 / 2} \exp \left\{-\frac{\epsilon-e \phi_{p}-\alpha_{p} \mu B_{p}}{\left(1-\alpha_{p}\right) T_{i}}\right\} \\
& =n_{i p} \sqrt{\frac{T_{i}}{2 \pi m_{i}}}\left(1-\alpha_{p}\right)\left[1-\frac{B_{m} / B_{p}-1}{B_{m} / B_{p}-\alpha_{p}} \exp \left\{-\frac{\mu_{*} B_{p}}{T_{i}}\right\}\right] \text {. }
\end{aligned}
$$

Here, $J_{i}^{+}$is the ion current coming from the central cell and escaping to end wall, $\widetilde{J}_{i}^{+}$is the ions of which are born around the plug and escape to the end wall, and $\widetilde{J}_{i}^{-}$is the current of ions that are born around the plug and escape to the central cell.

Noting that $\alpha_{p} \lesssim 1, \alpha_{q} \gtrsim 1$ and both constants $\alpha_{p}$ and $\alpha_{q}$ are close to unity, it is estimated that $\widetilde{J}_{i}^{+} / J_{i}^{+} \simeq\left(\alpha_{q}-1\right)$ and $\widetilde{J}_{i}^{-} / J_{i}^{+} \simeq\left(1-\alpha_{p}\right)$. All ions in a tandem mirror are produced by the ionization process of neutral atoms. The ratio of the ionization current around the plug (i.e., $\widetilde{J}_{i}^{+}+\widetilde{J}_{i}^{-}$) to the ionization current in the central cell (i.e., $J_{i}^{+}$) should be equal to the ratio $\left(\widetilde{J}_{i}^{+}+\widetilde{J}_{i}^{-}\right) / J_{i}^{+}$.

Assuming that the ionization occurs uniformly in a tandem mirror, the ratio $\left(\widetilde{J}_{i}^{+}+\widetilde{J}_{i}^{-}\right) / J_{i}^{+}$should be equal to the ratio of each volume $\left[\left(\Delta z / B_{p}\right) /\left(\int d z / B\right)\right]$, where $\Delta z$ is the width of plug and the integrations are carried out through the whole region in the tandem mirror.

The integration is estimated to be $\int_{0}^{z_{m}} d z / B \simeq 0.13 \mathrm{~cm} / \mathrm{G}$ in the GAMMA 10 standard magnetic field. Assuming $\Delta z=2$ $\mathrm{cm}$ and $B_{p}=10 \mathrm{kG}$, where $B_{p}$ is a fundamental electron cyclotron resonance point, the ratio becomes $\left(\Delta z / B_{p}\right) / \int(d z / B) \simeq 0.0015$. This value corresponds to that of $\alpha_{p} \simeq 0.998$ and $\alpha_{q} \simeq 1.0015$, in the parameters of which the continuous plug potential determined by the charge neutrality condition is created within the results of this manuscript.

As is seen in Figs. 11(b) and 11(d), the profile of the potential is very flat around the plug, so that the ions responsible for the distribution function characterized by $\alpha_{p}$ and $\alpha_{q}$ can be born in a wide flat area around the plug. The width $\Delta z$, therefore, can be larger than $2 \mathrm{~cm}$ in the GAMMA 10 tandem mirror, where the axial length of the end mirror cell is about $250 \mathrm{~cm}$.

The above estimation of the ionization rate neglects the plasma shielding of the plasma core from neutral gas. Shield- ing is significant in the central cell, but has a much smaller effect in the end mirror cell due to lower density. The gas pressure, however, is lower in the end mirror cell because the fueling sources are all in the central cell and the particle dumps around the end mirror cell may be well shielded from the plug region. The detailed analysis of the ionization rates around the end mirror cell, taking the above shielding into account, have not been done yet in the GAMMA 10 tandem mirror.

The position of the plug potential depends on the rate of ionization through the coefficient $\alpha_{p}$ in Eq. (4) as shown in Fig. 7(a). On the other hand, the electron cyclotron resonance point $\omega=\omega_{c e}$, where $\omega$ is the frequency of externally injected microwave, is located at $B / B_{b}=2$ in the GAMMA 10 tandem mirror. The efficiency of the plug potential formation, therefore, is expected to be best when the plug exists at $B / B_{b}=2$. Control of the ionization rate in the end mirror cell is important for plug potential formation.

The mechanism of a plug potential formation could be understood as follows: At first, we consider the situation without ECRH and ionization in the end mirror cell. The axial profile of the magnetic field is assumed to be symmetric around $z=z_{b}$, as shown in Fig. 12. The ion and electron orbits under consideration are the same as those in Fig. 2. The temperature of the electrons trapped in the region $z_{b}$ $\leqslant z \leqslant z_{m}$ is the same as that of the electrons passing from the central cell in Fig. 12(a), i.e., $\alpha_{e}=0$ in Eq. (14). Because the ion and electron densities and potential are a function of only $B$, with the assumption that the ion and electron distribution functions are constant along its orbits, the axial potential profile is symmetric around $z=z_{b}$, as shown in Fig. 12(a).

The asymmetry of the electron temperature around $z$ $=z_{b}$, i.e., $\alpha_{e} \neq 0$, can break the symmetry of the potential 

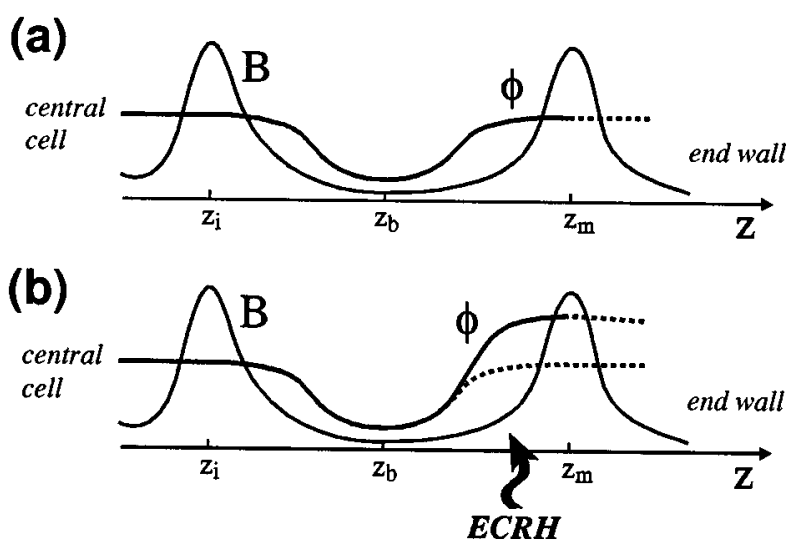

(c)

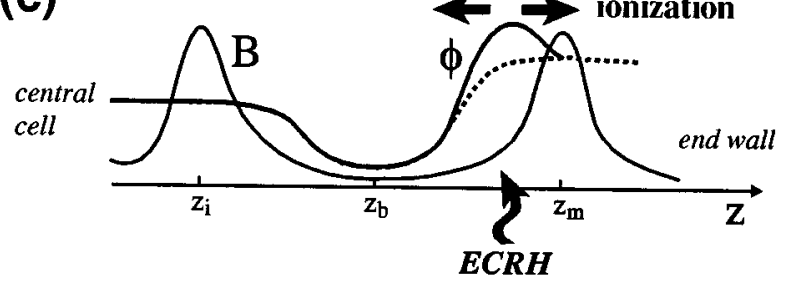

FIG. 12. Schematic diagram of axial potential profiles.(a) is the case without ECRH and ionization, (b) includes the ECRH but without ionization, and (c) includes both ECRH and ionization.

profile about $z=z_{b}$, which is shown in Fig. 12(b). If there is no ionization, i.e., $\alpha_{p}=0$, there is no solution satisfying $\partial n_{i} / \partial B_{p}=\partial n_{e} / \partial B_{p}$, that is $d \phi / d B \neq 0$ everywhere from $z$ $=z_{b}$ to $z=z_{m}$, as mentioned in Sec. III A. As indicated in Fig. 12(b), however, the maximum point of potential exists at $z=z_{m}$, because $d \phi / d z=(d \phi / d B)(d B / d z)$ and $d B / d z$ $=0$ at $z=z_{m}$.

The ionization has an effect on the location of the potential maximum and the height of the potential. The ions that are born around the plug region play a role like high-energy sloshing ions. When the plug potential becomes high enough to prevent the ions coming from the central cell from reaching the potential maximum, the ions that are born around the plug occupy a large part of the ion population there, and so, make the plug potential higher.

\section{SUMMARY}

We have shown that a plug potential can form even without the high-energy sloshing ions that are produced by neutral beam injection. The species required for the plug potential formation are the passing ions with Maxwellian, magnetically trapped ions with non-Maxwellian, passing electrons with Maxwellian and electrostatically trapped electrons with non-Maxwellian. A small amount of ions that are supplied around the plug is also required in order to avoid sheath formation. We suppose that those populations of ions and electrons are plausible in the present tandem mirror.

The parameter survey was carried out by using the distribution functions given in advance, the results of which revealed that the plug potential can form in a wide range of parameters of the distribution functions. The efforts to determine the distribution function itself self-consistently is required as a future problem. The essential conclusions, however, will not be too different from the present results.

The amount of supply of ions, which is required to form the plug potential with continuous axial profile, is not contradictory to that estimated in the GAMMA 10 parameter. This amount of the supply has an influence on the location of the plug potential, as well as the magnitude of the potential.

${ }^{1}$ G. I. Dimov, V. V. Zakaidakov, and M. E. Kishinevsky, Fiz. Plasmy 2, 597 (1976) [Sov. J. Plasma Phys. 2, 326 (1976)].

${ }^{2}$ T. K. Fowler and B. G. Logan, Comments Plasma Phys. Control. Fusion 2, 167 (1977).

${ }^{3}$ D. E. Baldwin and B. G. Logan, Phys. Rev. Lett. 43, 1318 (1979)

${ }^{4}$ R. F. Post, Nucl. Fusion 27, 1579 (1987).

${ }^{5}$ T. Tamano, Phys. Plasmas 2, 2321 (1995).

${ }^{6}$ R. S. Post, K. Brau, J. Casey, X. Chen, J. Coleman, H. R. Garner, M. Gerver, S. Golovato, D. Goodman, W. Guss, S. Hiroe, S. Hokin, S. Horne, J. Irby, J. Kesner, B. Lane, T. Morgan, L. Pócs, E. Sevillano, D. Smatlak, D. K. Smith, J. Sullivan, R. P. Torti, and X. Z. Yao, in Plasma Physics and Controlled Nuclear Fusion Research 1986, Proceedings of the 11th International Conference, Kyoto, 1986 (International Atomic Energy Agency, Vienna, 1987), Vol. 2, p. 251.

${ }^{7}$ I. Katanuma, Y. Kiwamoto, H. Washimi, K. Ishii, K. Yatsu, and S. Miyoshi, J. Phys. Soc. Jpn. 61, 542 (1992).

${ }^{8}$ I. Katanuma and J. A. Tataronis, J. Phys. Soc. Jpn. 62, 1209 (1993).

${ }^{9}$ Y. Tatematsu, Y. Kiwamoto, T. Saito, and T. Tamano, J. Phys. Soc. Jpn. 63, 558 (1994).

${ }^{10}$ I. Katanuma, Y. Kiwamoto, L. G. Bruskin, K. Yatsu, and T. Tamano, J. Phys. Soc. Jpn. 63, 393 (1994).

${ }^{11}$ Y. Kiwamoto, Y. Tatematsu, T. Saito, I. Katanuma, Y. Yoshimura, and T. Tamano, Phys. Plasmas 1, 3986 (1994).

${ }^{12}$ K. Ishii, Y. Maeda, K. Tsumori, Y. Shimouchi, I. Katanuma, A. Itakura, K. Yatsu, and S. Miyoshi, Phys. Fluids B 4, 3823 (1992).

${ }^{13}$ K. Ishii, M. Kotoku, T. Segawa, I. Katanuma, A. Mase, Y. Nakashima, and S. Miyoshi, Nucl. Fusion 30, 1051 (1990).

${ }^{14}$ K. Ishii, T. Tanaka, T. Nakada, T. Katori, A. Abe, T. Takahashi, I. Katanuma, A. Itakura, K. Yatsu, and T. Tamano, Jpn. J. Appl. Phys. 1 33, 5971 (1994).

${ }^{15}$ T. Saito, Y. Kiwamoto, Y. Tatematsu, Y. Yoshimura, T. Takahashi, M. Inutake, and T. Tamano, Phys. Plasmas 2, 352 (1995).

${ }^{16}$ I. Katanuma, K. Ishii, Y. Kiwamoto, T. Takahashi, T. Goto, Y. Tatematsu, T. Saito, K. Yatsu, and T. Tamano, Jpn. J. Appl. Phys. 1 34, 350 (1995).

${ }^{17}$ T. Saito, I. Katanuma, T. Aota, L. G. Bruskin, T. Cho, M. Hirata, H. Hojo, M. Ichimura, K. Ishii, A. Itakura, N. Katsuragawa, Y. Kiwamoto, J. Kohagura, A. Mase, Y. Nakashima, Y. Sakamoto, M. Shojil, T. Tamano, Y. Tatematsu, T. Tokuzawa, K. Yatsu, M. Yoshikawa, and Y. Yoshimura, in Fusion Energy Conference 1996, 11-17 October 1996, Paper No. IAEACN-64/C2-6, Proceedings of the 16th International Conference, Montréal, 1996 (International Atomic Energy Agency, Vienna, 1997).

${ }^{18}$ Y. Matsuda and T. D. Rognlien, Phys. Fluids 26, 2778 (1983).

${ }^{19}$ R. H. Cohen, Nucl. Fusion 21, 209 (1981).

${ }^{20}$ I. Katanuma, Y. Kiwamoto, Y. Tatematsu, K. Ishii, T. Saito, K. Yatsu, and T. Tamano, Phys. Plasmas 3, 2218 (1996). 